\title{
Legacy of Prof. Juraj Korpáš International Impact of Slovak School of Experimental Respirology
}

\author{
Jana PLEVKOVA, Ivan POLIACEK
}

Human health is the main role of medical research. Scientists were always intrigued by disease prevention, their diagnostics and proper treatment. In fact, research in medicine is always directed towards the improvement of the health care and improvement of the quality of life of the target population. Nowadays, physiological research, which is the base stone for clinical research, progresses fast forward, providing new information about body functions in health and diseases. This obvious progress is associated with modern methods, such as neuronal tracing, patch-clamp methods, electrophysiology, molecular biology and many more, which supported by comprehensive information technology guarantees high quality and complex data. Our younger colleagues, young scientists, post-docs or $\mathrm{PhD}$ students are well-trained and qualified in utilizing these new methods.

In this supplement, we decided with the kind permission of the editorial office of Physiological Research to flashback to the era of the research, which was driven mainly by the immense curiosity of scientists, supported by very little technology, custom-made equipment in old fashioned laboratories - yet providing important original information about body functions. Now and then. We want to point out and remind the tremendous efforts of our teacher prof. Juraj Korpáš DrSc., who was an amazing scientist and was able to collect comprehensive scientific information that is the base of our research activity nowadays. We want to share, how the legacy of our teacher is still alive and how we rely on his heritage and continue in research employing new methods, but keeping the same persistence and curiosity, as he did.

Prof. Juraj Korpáš DrSc. was the founder of Martin's School of Experimental Respirology - the Central European cradle for research of cough and other airway defensive reflexes, which is still the frontline idea of our research group. Researchers at the Jessenius Faculty of Medicine and physicians in the hospital here in Martin produce quality research and publish results with success in international journals. To remember the unique personality of prof. Juraj Korpáš DrSc. and his legacy we decided to highlight the research work of Martin's School of Experimental Respirology and collaborating research groups from France, the United States, United Kingdom, Italy, Poland and South Korea.

Prof. Juraj Korpáš DrSc. (1926 - 2009) was Slovak physician, doctor of general medicine who graduated at the Comenius University in Bratislava in 1951. Afterwards, he became an assistant professor at Medical School in Košice, where he was teaching pathological physiology. He was further promoted to associate professor in 1962 after successful habilitation. In 1966, prof. Korpáš has changed institution and moved to Martin, where he started to work as the head and the associate professor at the Department of pathological physiology of the Jessenius Faculty of Medicine. His inauguration to full professorship was finalized in 1972 at Masaryk University in Brno. His professional career continued until 2003 when he retired but still remained active at the department as the professor emeritus. He was always a massive source of support, invention and encouragement, which are dearly missed after his demise in 2009.

During professional career prof. Korpáš was a member of Scientific Councils at medical schools in Martin, Košice and Bratislava. He was also a member of national and international scientific societies and organizations (e.g. Slovak Medical Society, Slovak Society of Physiology and Pathology of Respiration, International Society for Pathophysiology, Czech and Hungarian Physiological Societies, Czech Medical Society of Jan Evangelista Purkyně and many more); he was awarded multiple distinguished awards and finalized numerous appointments of visiting scientists abroad in Hungary, England, Japan, Poland, India just to mention a few.

The most important contributions of prof. Korpáš to the scientific community are summarized in this 
paragraph, however, it is not possible to mention all of them. Prof. Korpáš described and defined the expiration reflex, which can be elicited from the vocal cords. Observation of his study group, which detected rapid expiratory response after the stimulation of vocal cords without preparatory inspiration, led to the characterization of expiration reflex not only the "fragment of cough reflex" as it was thought, but unique reflex with its afferent and efferent pathways. Expiration reflex and cough are separate reflexes, they have specific regulation, function and they differ e.g. in a response to antitussive drugs or anesthesia (Korpáš and Tomori 1979).

Prof. Korpáš also described conditions that lead to the augmentation and attenuation of cough responses these observations were published 42 years ago and were not reported in the literature until 2005 as "down- and upregulation of cough" (Korpáš and Widdicombe 2002).

He invented and introduced a unique method of tusiphonography. This method relies on recording and detection of the cough sound and its further analysis. This was a breakthrough idea to study cough sound in different pathological conditions in an attempt to find a specific pattern that would allow differentiation of diagnoses based on the cough sound. Team of prof. Korpáš developed multiple models of respiratory diseases in laboratory animals suitable for the study of cough. Together with prof. Tomori, he shares the description of the aspiration reflex (Korpáš and Paintal 2006). Prof. Korpáš with prof. Tomori built foundation of Martin's School of Experimental Respirology, which became known throughout the scientific world focused on respiratory problems, thanks to the conference Martin's Days of Respiration and regular publication of original knowledge.

Students and successors of professor Korpáš still organize Martin's Days of Respiration and continue to network with international research teams to deepen the knowledge on cough reflex, its regulations, pathologies and development of new diagnostic and therapeutic approaches. Articles published in this supplement are evidence of huge international and national cooperation of scientists involved in basic research as well as clinicians and other health care professionals in the field of the cough reflex.

Kunc and co-authors in their study addressed the changes in sensitivity of cough reflex in children with asthma. The question was whether prolonged airway inflammation, which is a characteristic feature of asthma, modulates neurophysiological properties of the airway afferent nerves mediating cough. The study performed in
25 children with asthma and 15 healthy controls revealed a statistically significant decrease of the threshold of airway afferents pointing towards neuroplastic changes of cough related afferents associated with asthma (Kunc et al. 2020a).

High prevalence of airway inflammatory diseases requires sophisticated non-invasive methods for monitoring of the severity of the pathological process, which can either indicate the progression of the disease or the recovery. While the measurement of exhaled NO (NO from the lower airways - FeNO) is already validated noninvasive technique for asthma monitoring, the monitoring of allergic rhinitis by measurement of nasal NO (nNO) has not been validated so far and its predictive value is not established at the moment. Next study tested levels of FeNO and nNO subjects with allergic rhinitis and healthy controls. Measurements were performed during the pollen season and out of it, and also before and after pharmacological treatment. The study found significant differences in FeNO and nNO between allergic subjects and controls at all set points of measurements. While values of FeNO in allergic subjects responded in an expected pattern to the pollen season and medication, the measurement of nNO does not seem to be optimal method for monitoring of severity of allergic inflammation in the upper airways and these tests must be interpreted carefully and in correlation with the symptom scores (Antosova et al. 2020).

Our supplement also contains a recent review of scientific papers focused on the identification of biomarkers which can predict asthma severity, the course of disease and response to treatment (Kunc et al. 2020b). Biomarker research has expanded greatly with the advancement of molecular research techniques. An ideal biomarker should be suitable to identify the disease as well as the specific endotype/phenotype, useful in the monitoring of the disease and to determine the prognosis with minimum discomfort or risk to the patient. The article suggests that an ideal biomarker doesn't exist at the moment because of "overlap" between the biomarkers, it is, however, possible to successfully identify individual endotypes of the diseases using panels of biomarkers in an era of molecular research techniques, precision and personalized medicine - which is the background for successful treatment.

Various non-pharmacological methods have been developed in respiratory physiology to alleviate respiratory symptoms, however their effectiveness if often questioned. Barski et al. (2020) studied the effectiveness 
of saline nebulization on lung functions in children suffering from different types of airway diseases. Measurements of lung functions were performed before and $15 \mathrm{~min}$ after saline nebulization in two designed groups - children with FEV1/FVC ratio below and above $80 \%$. The study found significant improvement after saline nebulization in FEV1, mid-expiratory flow at $50 \%$ and $75 \%$ of FVC and peak expiratory flow in the group with the baseline FEV1/FVC less than $80 \%$. Contrary to that, children with an index greater than $80 \%$ displayed no appreciable changes in the lung function variables. Authors concluded that isotonic saline nebulization might mitigate the functional signs of threatening pulmonary obstruction and as such may be clinically useful in pediatric patients with mild respiratory problems.

Non-pharmacological techniques are also employed in augmentation of cough. Contribution of an expert of British Thoracic Society Dr. Arietta Spinou highlights selected aspects of these techniques. Cough is an important mechanism of airway clearance and in patients who present with weak and ineffective cough, augmentation techniques aim to assist or simulate the maneuver. These techniques target different phases of the cough cycle, mainly the inspiratory and expiratory phases, through assisted inspiration, assisted expiration and their combination. They include the manual hyperinflation, ventilator hyperinflation, glossopharyngeal breathing, manually assisted cough and mechanical insufflatorexsufflator - each applied individually or in different combinations. The author provides an overview of the effectiveness and safety of these techniques. All commonly used techniques can theoretically improve airway clearance, as they generate higher cough peak flows compared to unassisted cough and associated adverse events are infrequent (Spinou 2020).

An excessive, irritable, productive or nonproductive coughing is associated with airway inflammation. A variety of mediators associated with airway inflammation overstimulate vagal airway fibers mediating cough, including C-fibers leading to hypersensitivity and hyperreactivity. Because current antitussives have limited efficacy and unwanted side effects, there is a continual demand for the development of a novel more effective antitussives for a new efficacious and safe cough treatment. Brozmanova and Pavelkova (2020) provide a review of novel treatment targets acting via inhibiting the activity of vagal $C$-fibers. They represent a rational approach to the development of effective antitussive drugs. Major attention is focused on blocking the generator potential associated with the specific ion channels. There is evidence that voltage-gated sodium channels $(\mathrm{NaVs}) \quad 1.7,1.8$ and 1.9 subtypes are predominantly expressed in airway cough-triggering nerves. Because NaVs are essential for action potentials initiation and conduction irrespective of the stimulus, $\mathrm{NaVs}$ became a promising neural target. New compounds should be selective and targeted at combating pathological cough associated with diseases while preserving the protective cough reflex.

One of the syndromes that manifest by excessive coughing is upper airway cough syndrome - previously named postnasal drip syndrome. It is one of the most common causes of chronic cough together with asthma and gastroesophageal reflux. The main mechanisms leading to cough in patients with diseases of the nose and sinuses are postnasal drip, direct irritation of nasal mucosa, inflammation in the lower airways, upper airway inflammation and the cough reflex sensitization. The cough present in this group of patients occurs probably due to hypersensitivity of the upper airways sensory nerve or lower airways sensory nerve, or a combination of both. Lučanská and co-authors reviewed current information about the pathogenesis of upper airway cough syndrome and state that further studies are necessary to elucidate this phenomenon to find not only symptomatic but also causal treatment (Lučanská et al. 2020).

Excessive coughing could be also a serious side effect of certain medications. Prof. Alyn Morice, the head of Cough Task Force of European Respiratory Society together with his co-authors discuss this problem. Since the recognition of angiotensin-converting enzyme inhibitors (ACEIs)-induced cough, the drug has been considered as a potential cause of chronic cough. This review presents recent knowledge on drug-induced coughs in patients with chronic cough. The paper focuses on ACEIs, for which there is a multitude of studies documenting their association with cough. Additional systemic drugs inducing cough as a side effect of treatment and the potential mechanisms are discussed (Shim et al. 2020).

Expiratory efforts, the prominent components of coughs, are produced due to the activation of the caudal ventral respiratory group (cVRG) expiratory premotoneurons (Korpáš and Tomori 1979). Cinelli and co-authors (Cinelli et al. 2020) discuss data suggesting that neurons located in the cVRG have an essential role in the generation of both the inspiratory and expiratory components of the cough reflex. Some lines of evidence 
indicate that cVRG expiratory neurons may subserve the alternation of inspiratory and expiratory cough bursts when strongly activated, possibly owing to the presence of axon collaterals. Of note, experimental findings such as blockade or impairment of glutamatergic transmission to the cVRG neurons lead to the view that neurons located in the cVRG are crucial for the production of the complete cough motor pattern. The involvement of bulbospinal expiratory neurons seems unlikely since their activation affects differentially expiratory and inspiratory muscles, while their blockade does not affect baseline inspiratory activity. Thus, other types of cVRG neurons with their medullary projections possibly contribute to the finetuning of the intensity of inspiratory and expiratory efforts. Central neuronal circuits regulating cough are very sophisticated. Interactions between different neuronal populations within the brainstem could be employed for the development of new treatment strategies, e.g. new antitussive drugs. The effects of GABA receptor agonists microinjections in medullary raphé on the mechanically induced tracheobronchial cough response in anaesthetized, un-paralyzed, spontaneously breathing cats was studied recently. The results suggest that GABA-ergic inhibition significantly contributes to the regulation of cough reflex by the action of both $\mathrm{GABA}_{\mathrm{A}}$ and $\mathrm{GABA}_{\mathrm{B}}$ receptors. The data are consistent with the inhomogeneous occurrence of GABA-ergic neurons in medullary raphé and their different involvement in the cough reflex control. Cells within rostral nucleus raphé obscure with the dominant role of $\mathrm{GABA}_{\mathrm{A}}$ receptors and neurons of rostral nucleus raphé pallidus and caudal nucleus raphé magnus with the dominant role of $\mathrm{GABA}_{\mathrm{B}}$ receptors participate in the regulation of cough expiratory efforts (Martvoň et al. 2020). All of these experiments are performed with the aim to provide new information for the development of pharmacological cough suppression strategies.

Defensive reflexes that clear airways and lungs are efficiently induced by the mechanical stimuli. It is speculated that provocation of defensive response depends not only on stimulus duration but also on stimulus velocity. Authors from the University of Lorraine, France performed a set of experiments on anaesthetized and tracheotomized rabbits to address this question. Mechanical stimulus was provoked by a mechanical probe introduced through the tracheotomy and rotated by a small electrical motor using a rotational velocity of $40 \mathrm{rpm} / \mathrm{s}$ and $20 \mathrm{rpm} / \mathrm{s}$. Threshold, incidence and intensity of cough reflex were analyzed for each animal. The findings of this study are of interest as they show that protective reflex cough, clearing airways even during sleep or anesthesia, is tuned by mechanical stimulus velocity (Demoulin et al. 2020).

Laboratory research of cough reflex is a very important source of clinically relevant information in every field of biomedical research. However, in the study of airway defensive reflexes researchers utilize almost exclusively male guinea pigs. It represents a significant obstacle in the successful translation of results into clinical practice because, for example, chronic hypersensitivity cough syndrome, affects mostly postmenopausal women. No cause for such exaggerated cough can be found, therefore this condition cannot be treated appropriately. One of the reasons leading to the lack of relevant data about mechanisms responsible for hypersensitivity of cough-related pathways is gender bias, which is present in nearly all branches of biomedical research. Recent study focuses on detailed characterization of cough response to aerosols of commonly used tussive agents (capsaicin, distilled water, allyl isothiocyanate, cinnamaldehyde and citric acid) in female guinea pigs. In pooled data from multiple challenges, no statistical difference in the number of coughs and cough latency between sexes were found. Autors concluded that the female guinea pig model is as useful as the male model, and both sexes of guinea pigs are equally suitable for experimental cough studies (Sterusky et al. 2020).

Martin's group of experimental respirology participates on the research projects, which integrate respiratory physiology with cardiovascular physiology. A bidirectional relationship between cough and heart dysfunctions are presented in the contribution of Grabczak and co-authors, with the special insights into an arrhythmia-triggered cough. Albeit rare, cough induced by cardiac pathologies (mainly arrhythmias) seems to be an interesting and underestimated phenomenon. This condition is usually associated with the presence of abnormal heart rhythms and ceases with successful treatment of arrhythmia either by pharmacotherapy or by radiofrequency ablation of arrhythmogenic substrate. The two main hypotheses on cough-heart relationships - reflex and hemodynamic - are discussed in the review, including the authors' perspective based on the experiences with an arrhythmia-triggered cough (Grabczak et al. 2020).

An excellent demonstration of cardio-respiratory interactions is respiratory sinus arrhythmia (RSA), i.e. heart rate (HR) variations during inspiration and expiration. Variations of heart rate is considered as a non-invasive index of cardiac vagal control, pointing towards increased 
cardiovascular risk of it is not normal. Mitral valve prolapse (MVP) could be associated with increased cardiovascular risk; however, the studies are rare particularly at adolescent age. A recent study performed in adolescent girls revealed reduced RSA magnitude indicating impaired cardiac vagal control in MVP already at adolescent age that could be crucial for early diagnosis of cardiovascular risk in MVP. This conclusion was set based on analysis of EKG records (Bona Olexova et al. 2020).

Interdisciplinary research cooperation of Martin's group of experimental respirology with the Clinic of Dermatology produced interesting findings in the past (Pecova et al. 2003a, Pecova et al. 2003b). A recent contribution to the special issue points towards differences and similarities between cough afferents and afferent nerves mediating itch. Itch is the most common chief complaint in patients visiting dermatology clinics. It is analogous to cough and sneeze stimuli perception of the lower and upper respiratory tract, all three of which are host actions trying to clear noxious stimuli. The itch can originate from a variety of etiologies. Itch originates following the activation of peripheral sensory nerve endings following damage or exposure to inflammatory mediators. More than one sensory nerve subtype is thought to serve pruriceptive itch which includes both unmyelinated C-fibers and thinly myelinated $\mathrm{A} \delta$ nerve fibers. There are a lot of mediators capable of stimulating these afferent nerves resulting in itching. Cough and itch pathways are mediated by small-diameter sensory fibers. These cough and itch sensory fibers release neuropeptides upon activation, which leads to inflammation of the nerves. The inflammation is involved in the development of chronic conditions of itch and cough. The aim of this review is to point out the role of sensory nerves in the pathogenesis of cough and itching. The common aspects of itch and cough could lead to new thoughts and perspectives in both fields (Pecova et al. 2020).

Beside the reflex protective and defensive reflexes professor Korpáš always pointed towards the importance of their cooperation and complementary role to the non-reflex protective and defensive mechanisms, which operate in the airways at all times. The sinonasal mucosa has an essential role in defensive mechanisms of the upper respiratory tract. The innate immune system presents the primary defense against noxious microorganisms followed by induction of the adaptive immune mechanisms as a consequence of the presence of pathogens. This well-known activation of the adaptive immune system in response to the presence of the antigen on mucosal surfaces is now broadly applied in vaccinology research. Prevention of infectious diseases belongs to substantial challenges in maintaining population health. Non-invasive, easily applicable mucosal vaccination purposes various research opportunities that could be usable in daily practice. However, the existence of multiple limitations such as rapid clearance of vaccine from nasal mucosa by means of mucociliary transport represents a great challenge in the development of safe and efficient vaccines. Pedan and co-authors (Pedan et al. 2020) provide a comprehensive updated view on nasal functions with the focus on nasal mucosal immunity and its potential application in vaccination in the near future.

Airway defensive reflexes require powerful expiration to clean the airways. In subjects with neuromuscular diseases, however, there is a problem with their execution. Parkinson's disease (PD) is most commonly manifested by the presence of motor symptoms - one of them is attenuated cough strength and effectiveness of this reflexive motor behavior. However, non-motor symptoms occur several years before the onset of motor symptoms themselves. Hallmarks of dysfunction of the respiratory system are still outside the main focus of interest of either clinicians or scientists, despite their indisputable contribution to the morbidity and mortality of patients suffering from PD. In addition, many of the respiratory symptoms are already present in the early stages of the disease and efforts to utilize these parameters in the early diagnosis of PD are now intensifying. Mechanisms that lead to the development and progression of respiratory symptoms are analyzed in the review by Pokusa and co-authors. This review focuses mainly on the comparison of respiratory problems observed in clinical studies with available findings from experimental animal models. It also explains pathological changes observed in non-neuronal tissues in subjects with PD (Pokusa et al. 2020).

We are proud to publish contributions by the scientists from "Slovak group of experimental respirology" along with the articles of their colleagues from abroad. Thanks to the contributors, we were fortunate to receive numerous high-quality submissions that represent the scope of currently ongoing research which takes into account different aspects of cough.

Jana Plevkova Ivan Poliacek Guest Editors 


\section{References}

ANTOSOVA M, BENCOVA A, MOKRA D, PLEVKOVA J, PEPUCHA L, BUDAY T: Exhaled and nasal nitric oxide - impact for allergic rhinitis management. Physiol Res 69 (Suppl 1): S123-S130, 2020.

BARSKI P, SURDACKI M, SAJ A, WRÓBLEWSKA A, ORNAT M, PAWELAK A, POMPA D, JURGIEL J, ERMISCH V, HIRNLE A, PIROGOWICZ I, STANISŁAWSKA I, ŁYP M, POKORSKI M: Isotonic saline nebulization and lung function in children with mild respiratory ailments. Physiol Res 69 (Suppl 1): S131-S137, 2020.

BONA OLEXOVA L, SEKANINOVA N, JURKO A JR, VISNOVCOVA Z, GRENDAR M, JURKO T, TONHAJZEROVAI: Respiratory sinus arrhythmia as an index of cardiac vagal control in mitral valve prolapse. Physiol Res 69 (Suppl 1): S163-S169, 2020.

BROZMANOVA M, PAVELKOVA N: The prospect for potent sodium voltage-gated channel blockers to relieve an excessive cough. Physiol Res 69 (Suppl 1): S7-S18, 2020.

CINELLI E, IOVINO L, BONGIANNI F, PANTALEO T, MUTOLO D: Essential role of the cVRG in the generation of both the expiratory and inspiratory components of the cough reflex. Physiol Res 69 (Suppl 1): S19-S27, 2020.

DEMOULIN B. COUTIER-MARIE L, IOAN I, SCHWEITZER C, FOUCAULD L, DEMOULIN-ALEXIKOVA S: In vivo documentation of stimulus velocity tuning of mechanically induced reflex cough. Physiol Res 69 (Suppl 1): S139-S145, 2020.

GRABCZAK EM, STEC S, DABROWSKA M, PLEVKOVA J, KRENKE R: Cough as a cause and consequence of heart dysfunction - current state of art. Physiol Res 69 (Suppl 1): S105-S121, 2020.

KORPAS J, PAINTAL AS, ANAND A: Cough. From lab to clinic. Ane Books India, New Delhi, 2006, 348 p.

KORPAS J, TOMORI Z: Cough and Other Respiratory Reflexes. Progress in Respiration Research, Vol. 12, H HERZOG (ed), Karger, Basel, 1979, 356 p.

KORPAS J, WIDDICOMBE JG (eds): Cough. Recent Advances in Understanding. European Respiratory Review 12 (85). Procter and Gamble, Montpellier, France, 2002, 282 p.

KUNC P, FABRY J, LUCANSKA M, PECOVA R: Biomarkers of bronchial asthma. Physiol Res 69 (Suppl 1): S29-S34, 2020.

KUNC P, FABRY J, LUCANSKA M, ZATKO T, GRENDAR M, PECOVA R: Cough reflex sensitivity in asthmatic children. Physiol Res 69 (Suppl 1): S147-S150, 2020.

LUCANSKA M, HAJTMAN A, CALKOVSKY V, KUNC P, PECOVA R: Upper airway cough syndrome in pathogenesis of chronic cough. Physiol Res 69 (Suppl 1): S35-S42, 2020.

MARTVON L, KOTMANOVA Z, DOBROLUBOV B, BABALOVA L, SIMERA M, VETERNIK M, PITT T, JAKUS J, POLIACEK I: Modulation of cough reflex by GABA-ergic inhibition in medullary raphé of the cat. Physiol Res 69 (Suppl 1): S151-S161, 2020.

PECOVA R, FRLICKOVA Z, PEC J, TATAR M: Cough sensitivity in atopic dermatitis. Pulm Pharmacol Ther 16: $203-$ $206,2003$.

PECOVA R, FRLICKOVA Z, PEC J, TATAR M: Cough sensitivity in localized scleroderma with no clinical symptoms from lower airways. J Physiol Pharmacol 54 (Suppl 1): 25-28, 2003.

PECOVA T, KOCAN I, VYSEHRADSKY R, PECOVA R: Itch and cough - similar role of sensory nerves in their pathogenesis. Physiol Res 69 (Suppl 1): S43-S54, 2020.

PEDAN H, JANOSOVA V, HAJTMAN A, CALKOVSKY V: Non-reflex defense mechanisms of upper airway mucosa: possible clinical application. Physiol Res 69 (Suppl 1): S55-S67, 2020.

POKUSA M, HAJDUCHOVA D, BUDAY T, KRALOVA TRANCIKOVA A: Respiratory function and dysfunction in Parkinson-type neurodegeneration. Physiol Res 69 (Suppl 1): S69-S79, 2020.

SHIM JS, SONG WJ, MORICE AH: Drug-induced cough. Physiol Res 69 (Suppl 1): S81-S92, 2020.

SPINOU A: A review on cough augmentation techniques: assisted inspiration, assisted expiration and their combination. Physiol Res 69 (Suppl 1): S93-S103, 2020.

STERUSKY M, PLEVKOVA J, GRENDAR M, BUDAY T: Female guinea pig model for cough studies and its response to most common tussive substances. Physiol Res 69 (Suppl 1): S171-S179, 2020. 